\title{
Probing light dark sectors with coherent neutrino-nucleus scattering
}

\section{Adam Ritz*}

Department of Physics and Astronomy, University of Victoria, Victoria, BC V8P 5C2, Canada

E-mail: aritz@uvic.ca

\begin{abstract}
Experiments aiming to detect coherent neutrino-nucleus scattering present opportunities to probe new light weakly-coupled states, such as sub-GeV mass dark matter, in several extensions of the Standard Model. These states can be produced along with neutrinos in the collisions of protons with the target, and their production rate can be enhanced if there exists a light mediator produced on-shell. We consider the sensitivity reach of the COHERENT experiment at SNS, which recently observed coherent neutrino-nucleus scattering, to light dark matter interacting with the Standard Model via a light vector mediator coupled to the electromagnetic current.
\end{abstract}

The 19th International Workshop on Neutrinos from Accelerators-NUFACT2017

25-30 September, 2017

Uppsala University, Uppsala, Sweden

${ }^{*}$ Speaker. 


\section{Introduction}

The cosmic neutrino background constitutes an example of relic dark matter (DM). Although relativistic at decoupling, and playing a sub-dominant role in structure formation, neutrinos are otherwise characteristic of a cosmic relic that can undergo elastic scattering with nuclei. While the small mass and low temperature of the cosmic neutrino background makes this scattering channel a very challenging target for detection, the signature of coherent neutrino-nucleus scattering served as the initial template for the signatures used in underground dark matter detectors [1].

Coherent elastic neutrino-nucleus scattering ( $\mathrm{CEvNS})$ is, of course, of considerable interest in its own right. An accurate determination of the cross section may allow an independent measurement of the low-energy value of the weak mixing angle $\theta_{W}$. Moreover, deviations may signal physics beyond the Standard Model (SM) in the neutrino sector, often parametrized as NSI - nonstandard neutrino interactions (see e.g. [2]). The experimental activity directed towards CEvNS has intensified recently, focusing on stopped-pion source of neutrinos [3], and this year the COHERENT experiment at the SNS announced the first direct detection of CEvNS [4].

With the close analogy between CEvNS and dark matter scattering in mind, it should not be a surprise that proposals to measure coherent neutrino scattering in high luminosity fixed target experiments are also well suited to searching for new light states other than the SM neutrinos. Here we review some work [5], showing that modern fixed target proposals designed to observe and measure CEVNS are also sensitive to generic models of light dark matter in the $1-100 \mathrm{MeV}$ mass range. More generally, the use of high intensity proton-beam fixed target neutrino oscillation experiments $[6,7,8,9]$ and also electron-beam fixed target experiments (see e.g. [10, 11] for recent reviews) has recently been highlighted as a means to probe the light dark matter parameter space, and a dedicated beam-dump run was recently carried out at the MiniBooNE experiment $[12,13]$. In order not to over-produce light dark matter in the early universe, consistent models generically require a relatively light force carrier mediating the interaction between the SM and dark sector, and providing an efficient annihilation channel [14]. The light mass scale of the mediator, in turn, increases the production rate of light DM in fixed target collisions.

In the remainder of this contribution, we will review the sensitivity of COHERENT and similar facilities to light sub-GeV dark matter following [5], updating those results with the recent direct CEvNS measurement. We will focus on a simple benchmark light dark matter model, with a light kinetically-mixed dark photon [15]. This class of models is well motivated on effective field theory grounds since a kinetically mixed vector is one of the few renormalizable portal couplings to a neutral hidden sector. In the following sections we define this model more precisely, and then analyze the sensitivity of CEvNS experiments to production of the vector mediator in the target through both pion decay in flight and charged pion capture, with subsequent on- or off-shell decay to dark matter which then scatters coherently (or incoherently) off nuclei in the target.

\section{Light dark states}

Light thermal relic dark matter, with a mass below a few $\mathrm{GeV}$, generically requires new annihilation channels with light mediators in order not to over-close the universe. The simplest mediators couple via the renormalizable portal interactions. 


\subsection{Benchmark model for light dark matter}

The model we study uses a spontaneously broken $\mathrm{U}(1)^{\prime}$ gauge symmetry in the hidden sector, leading to a massive vector $V_{\mu}$ which is kinetically mixed with the photon [16], and dark matter is a hidden scalar or fermion $\chi$ charged under $U(1)^{\prime}$. At low energies, the Lagrangian is given by

$$
\mathscr{L}=\mathscr{L}_{\chi}-\frac{1}{4} V_{\mu v} V^{\mu v}+\frac{1}{2} m_{V}^{2} V_{\mu} V^{\mu}-\frac{\kappa}{2} V^{\mu v} F_{\mu v}+\cdots
$$

with

$$
\mathscr{L}_{\chi}= \begin{cases}i \bar{\chi} \not D \chi-m_{\chi} \bar{\chi} \chi, & (\text { Dirac fermion DM }) \\ \left|D_{\mu} \chi\right|^{2}-m_{\chi}^{2}|\chi|^{2}, & (\text { Complex scalar DM })\end{cases}
$$

where $D=\partial-i g^{\prime} q_{e} V$, with $g^{\prime}\left(q_{e}\right)$ the $U(1)^{\prime}$ gauge coupling (charge), and the ellipsis denotes terms associated with the spontaneous breaking of $U(1)^{\prime}$, which will not be important here.

While we will consider kinematics with both on- and off-shell mediators, it is important to note that when the vector $V$ can decay on-shell to dark matter, $m_{V}>2 m_{\chi}$, a light complex scalar DM candidate (which we shall focus on) is less constrained by the impact of annihilation on the $\mathrm{CMB}$, as it is $p$-wave suppressed.

\section{Production and detection of light states}

\subsection{Fixed target production modes}

Having in mind the relatively low beam energy at the SNS, we will account for a number of production modes from meson decay.

(i) $\pi^{0}$ decay in flight:- A dominant production mode in the forward direction utilizes radiative $\pi^{0}$ decay,

$$
\pi^{0} \longrightarrow \gamma+V^{(*)} \longrightarrow \gamma+\chi^{\dagger}+\chi .
$$

We allow for on- or off-shell $V^{*} \rightarrow \chi^{\dagger} \chi$ decays, with

$$
\Gamma_{\pi^{0} \rightarrow \gamma \chi^{\dagger} \chi}=\frac{1}{4 \pi m_{\pi}} \int d \Pi_{\pi^{0} \rightarrow \gamma V} d \Pi_{V \rightarrow \chi^{\dagger} \chi} d q^{2}|\mathscr{M}|^{2}, \quad|\mathscr{M}|^{2}=\frac{\kappa^{2} \alpha^{\prime} \alpha^{2} f\left(q^{2}, p \cdot k_{1}, p \cdot k_{2}\right)}{\pi f_{\pi}^{2}\left[\left(q^{2}-m_{V}^{2}\right)^{2}+m_{V}^{2} \Gamma_{V}^{2}\right]},
$$

where $f=\left(q^{2}-4 m_{\chi}^{2}\right)\left(m_{\pi}^{2}-q^{2}\right)^{2}-4 q^{2}\left(p \cdot k_{1}-p \cdot k_{1}\right)^{2}$. Here $p / q$ are the photon/ $V$ momenta, and $k_{1,2}$ are the momenta of the dark sector particles in the final state, so that $q=k_{1}+k_{2}$.

(ii) $\pi^{-}$capture:- In addition to radiative pion decays, an isotropic production mode involves $\pi^{-}$ capture on protons, through a version of the Panofsky process

$$
\pi^{-}+p \longrightarrow n+V^{(*)} \longrightarrow n+\chi^{\dagger}+\chi .
$$

This mode provides an approximately monochromatic source of $V$, which gives an isotropic source of dark matter with a 'rectangular' energy distribution in the lab frame. It is particularly relevant for detectors located at large angles relative to the beam axis, as is the case for the CEVNS proposals. We utilize data from [17], which shows a sharp radiative peak with energy $E \sim 130 \mathrm{MeV}$. 
A Monte Carlo code is used to calculate the angular acceptance and energy distribution of the dark matter particles generated through the previously discussed production channels. The $\pi^{0}$ production distribution, assumed to be well approximated by an average of the $\pi^{+}$and $\pi^{-}$ Burman-Smith distributions, is sampled to generate $\pi^{0}$ decays in flight. The number of charged pions produced at the SNS is: $N_{\pi^{-}}=0.05 \times$ p.o.t, $N_{\pi^{+}}=0.17 \times$ p.o.t. [3], where the number of protons on target (p.o.t.) for a year's running is $\sim 10^{23}$.

\subsection{Nuclear Scattering Rate}

Depending on the momentum exchanged, scattering off nuclei can be either coherent or incoherent. Given that coherent scattering dominates for low momentum transfer, it is simplest to model the transition with a form-factor as is used in direct detection.

For incoherent scattering off nucleons, the leading term in the cross section has the form,

$$
\frac{d \sigma_{\chi N}}{d E_{\chi}}=4 \pi \kappa^{2} \alpha \alpha^{\prime} q_{e}^{(N)} G_{D}\left(Q^{2}\right) \frac{2 m_{N} E E_{\chi}-m_{\chi}^{2}\left(E-E_{\chi}\right)}{\left(E^{2}-m_{\chi}^{2}\right)\left(m_{V}^{2}+Q^{2}\right)}+\cdots
$$

where $E_{\chi}$ is now the energy of the recoiling DM particle, while $Q^{2}=2 m_{N}\left(E-E_{\chi}\right)$ is the momentum transfer. $G_{D}\left(Q^{2}\right)$ the Sachs form-factor, and $q_{e}^{(N)}$ the electric charge of the nucleon $N=p, n$. The ellipsis denotes terms associated with the nucleon dipole form-factors, which are generally subleading (for protons) and are neglected here to simplify the presentation. However, the full results incorporating these extra terms are given in $[7,18]$, and are included in the numerical analysis below.

Of particular interest in the present analysis is the possibility of coherent scattering off nuclei in the detector, for light vector masses. We can write the overall nuclear scattering cross section in the form

$$
\sigma_{\chi \mathrm{A}}(E)=\int_{E_{\chi}^{\min }}^{E_{\chi}^{\max }} d E_{\chi}\left(f_{p}\left(q^{2}\right) \frac{d \sigma_{\chi p}}{d E_{\chi}}+f_{n}\left(q^{2}\right) \frac{d \sigma_{\chi n}}{d E_{\chi}}\right)
$$

where $q=\sqrt{2 M\left(E-E_{\chi}\right)}$ is the nuclear recoil momentum, with $E$ the initial kinetic energy. The form factors $f_{N=p, n}\left(q^{2}\right)=n_{N}$ for $q^{2}>(50 \mathrm{MeV})^{2}$, and we utilize the Helm form-factor at small momentum transfer, so that $f_{N=p, n}\left(q^{2}\right)=n_{N}^{2}\left|F_{\mathrm{Helm}}\left(q^{2}\right)\right|$ for $q^{2}<(50 \mathrm{MeV})^{2}$, where $n_{p}=Z, n_{n}=$ $(A-Z)$ for $\mathrm{U}(1)^{\prime}$, in terms of the atomic number and mass of the nucleus.

We initially consider a hypothetical detector geometry for COHERENT: A cylindrical 1 tonne CsI detector located twenty meters away from the target at a right angle to the beam-line direction. We also consider the current experimental setup with a $14.6 \mathrm{~kg}$ detector situated in the "neutrino alley". The expected scattering signal was calculated by iterating over all dark matter 4-momenta generated by the production Monte Carlo that intersect the detector. Energies for recoiling nuclei are selected by sampling (5), and those that do not pass the kinematic cuts chosen for each experiment are discarded. Minimum recoil energy cuts were chosen such that the prompt neutrino background is minimized. For COHERENT, a cut of $E_{\text {recoil }}>16 \mathrm{keV}$ was adopted. Incoherent nucleon scattering was also considered for $q^{2}>(50 \mathrm{MeV})^{2}$, and its contribution to the total signal was negligible for $m_{V}<m_{\pi^{0}}$. The total number of signal events can be written as

$$
N_{A \chi \rightarrow A \chi}=n_{A} \varepsilon_{\text {eff }} \sum_{\substack{\text { prod. } \\ \text { chans. }}}\left(\frac{N_{\chi}}{2 N_{\text {trials }}} \sum_{i} L_{i} \sigma_{A \chi, i}\right),
$$



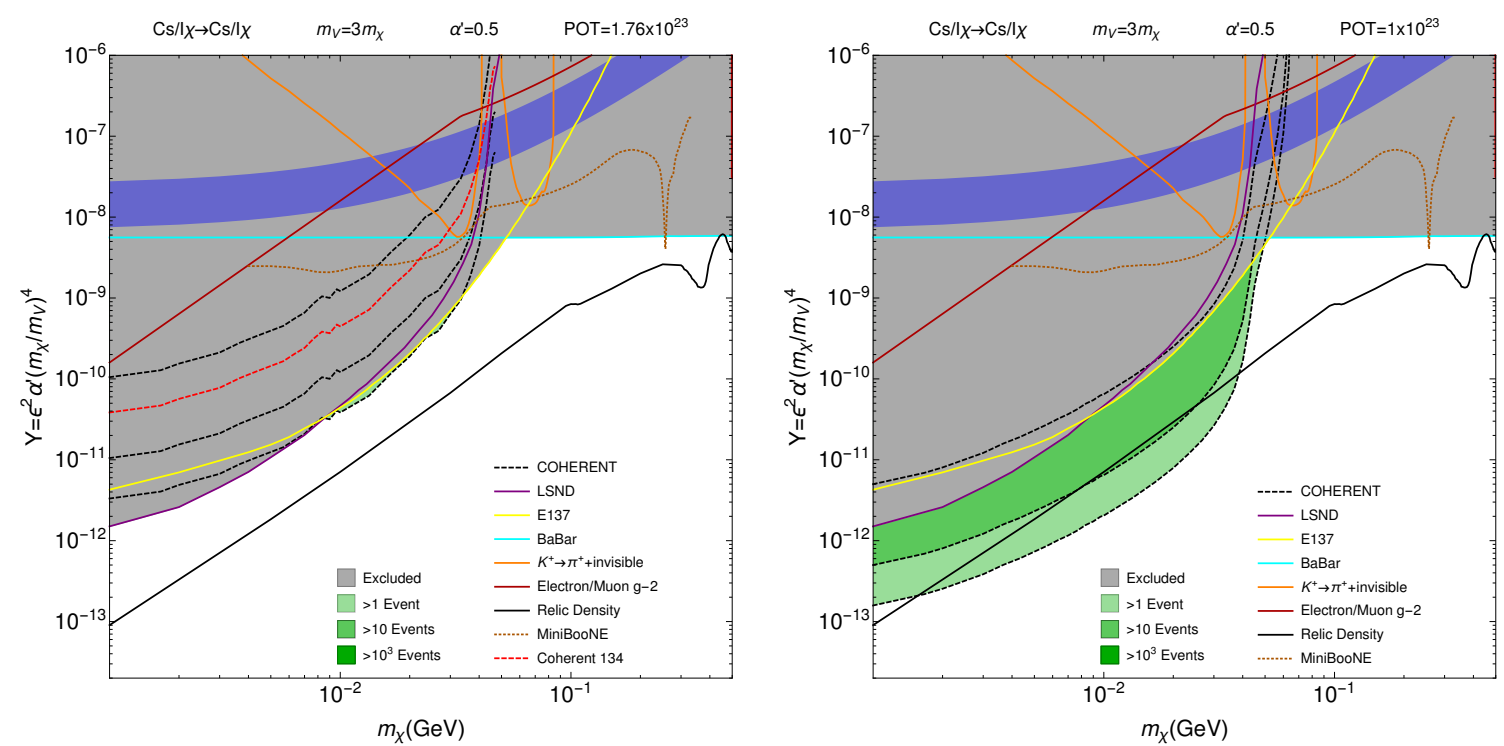

Figure 1: Sensitivity contours for scattering events at COHERENT (SNS), with the three green-shaded contour regions corresponding to 1 event (light), 10 events (medium) and 1000 events (dark). On the left, we show the sensitivity with the current $14.6 \mathrm{~kg}$ detector (the 1-event contour just crosses the existing limit from LSND), and on the right the projected sensitivity with a 1 tonne CsI detector. In grey are exclusions from other sources (see [5, 9] details), and the black contour indicates the parameters required to ensure the correct dark matter relic density.

where $A=\mathrm{Li}, \mathrm{Cs}$, and $\varepsilon_{\text {eff }}$ is the detection efficiency for events within the detector volume (we assume 50\%). The inner sum is over all dark matter 4-momenta $p_{i}$ generated by the production Monte Carlo, $L_{i}$ is the length of the intersection between the dark matter trajectory (with momentum $\left.p_{i}\right)$ and the detector ( $L_{i}=0$ if the trajectory does not pass through the detector), $\sigma_{A \chi}$ is the scattering cross section (5) between $\chi$ and the nucleus $A, N_{\text {trials }}$ is the total number of decays generated by the production Monte Carlo and $N_{\chi}=2 N_{\pi^{0}, \pi^{-}} \operatorname{Br}\left(\pi^{0}, \pi^{-} \rightarrow \chi \chi^{\dagger} X\right)$ is the number of dark matter particles produced, where $X$ includes any non-hidden sector end products.

\section{Sensitivity at SNS}

We present the results for COHERENT in Fig. (1), showing sensitivity contours corresponding to 1,10 and 100 events. On the left, we update the results of [5], showing the projected sensitivity with a hypothetical 1 tonne CsI detector, which is impressive, while on the right we show the actual sensitivity of the current experiment with a $14.6 \mathrm{~kg}$ detector. The latter contours suggest sensitivity slightly below that of experiments such as LSND to models in this class, but it is important to bear in mind that those experiments rely on electron scattering, while COHERENT only utilizes the coupling to nucleons. The computation uses cuts on the recoil energy spectrum designed to remove the coherent neutrino scattering background, and thus we anticipate that the actual sensitivity should be quite good, potentially at the $\mathscr{O}(\mathrm{few})$ event level. However, a full analysis would be required to determine the full background in more detail.

This sensitivity to light dark matter provides an added motivation to pursue CEvNS experiments of this kind, beyond those associated with detecting and precisely measuring the coherent neutrino-nucleus scattering cross section. 
Acknowledgements: I'd like to thank the organizers for their invitation to NUFACT, and my coauthors, P. deNiverville and M. Pospelov, for a fruitful and enjoyable collaboration on the work described here.

\section{References}

[1] M. W. Goodman and E. Witten, Detectability of Certain Dark Matter Candidates, Phys.Rev. D31 (1985) 3059.

[2] K. Scholberg, Prospects for measuring coherent neutrino-nucleus elastic scattering at a stopped-pion neutrino source, Phys.Rev. D73 (2006) 033005, [hep-ex/ 0511042$].$

[3] J. Collar, N. Fields, M. Hai, T. Hossbach, J. Orrell et al., Coherent neutrino-nucleus scattering detection with a CsI[Na] scintillator at the SNS spallation source, 1407.7524.

[4] COHERENT collaboration, D. Akimov et al., Observation of Coherent Elastic Neutrino-Nucleus Scattering, Science 357 (2017) 1123-1126, [1708.01294].

[5] P. deNiverville, M. Pospelov and A. Ritz, Light new physics in coherent neutrino-nucleus scattering experiments, Phys. Rev. D92 (2015) 095005, [1505.07805].

[6] B. Batell, M. Pospelov and A. Ritz, Exploring Portals to a Hidden Sector Through Fixed Targets, Phys.Rev. D80 (2009) 095024, [0 906.5614 ].

[7] P. deNiverville, M. Pospelov and A. Ritz, Observing a light dark matter beam with neutrino experiments, Phys.Rev. D84 (2011) 075020, [1107.4580].

[8] P. deNiverville, D. McKeen and A. Ritz, Signatures of sub-GeV dark matter beams at neutrino experiments, Phys.Rev. D86 (2012) 035022, [1205.3499].

[9] P. deNiverville, C.-Y. Chen, M. Pospelov and A. Ritz, Light dark matter in neutrino beams: production modelling and scattering signatures at MiniBooNE, T2K and SHiP, Phys. Rev. D95 (2017) 035006, [1609.01770].

[10] J. Alexander et al., Dark Sectors 2016 Workshop: Community Report, 2016, 1608.08632 , http://inspirehep.net/record/1484628/files/arXiv:1608.08632.pdf.

[11] M. Battaglieri et al., US Cosmic Visions: New Ideas in Dark Matter 2017: Community Report, 1707.04591.

[12] MiniBoone Collaboration collaboration, R. Dharmapalan et al., Low Mass WIMP Searches with a Neutrino Experiment: A Proposal for Further MiniBooNE Running, 1211.2258.

[13] MiniBoonE collaboration, A. A. Aguilar-Arevalo et al., Dark Matter Search in a Proton Beam Dump with MiniBooNE, Phys. Rev. Lett. 118 (2017) 221803, [1702 . 02688].

[14] C. Boehm and P. Fayet, Scalar dark matter candidates, Nucl.Phys. B683 (2004) 219-263, [hep-ph/0305261].

[15] M. Pospelov, A. Ritz and M. B. Voloshin, Secluded WIMP Dark Matter, Phys.Lett. $B 662$ (2008) 53-61, [0711.4866].

[16] B. Holdom, Two U(1)'s and Epsilon Charge Shifts, Phys.Lett. B166 (1986) 196.

[17] R. MacDonald, D. Beder, D. Berghofer, M. Hasinoff, D. F. Measday et al., Charge Exchange of Stopped pi- in Deuterium: Experiment and Theory, Phys.Rev.Lett. 38 (1977) 746.

[18] B. Batell, P. deNiverville, D. McKeen, M. Pospelov and A. Ritz, Leptophobic Dark Matter at Neutrino Factories, 1405.7049. 